\title{
3 Research Square \\ Expedient Standing Or Squatting Placental Delivery And Postpartum Hemorrhage: A Retrospective Cohort Study
}

Judy Slome Cohain ( $\sim$ judyslome@hotmail.com )

Hadassah Ein Kerem Medical Center

Rina E. Buxbaum

Hadassah Ein Kerem Medical Center

Research Article

Keywords: Postpartum hemorrhage, Maternal mortality, Maternal morbidity, Third stage management

Posted Date: January 31st, 2022

DOI: https://doi.org/10.21203/rs.3.rs-1265252/v1

License: (c) (i) This work is licensed under a Creative Commons Attribution 4.0 International License.

Read Full License 


\section{Abstract}

Objective: To compare expedient delivery of the placenta in standing or squatting by 3 minutes postpartum to current third stage management.

Design, Setting, Sample and Methods: A retrospective cohort study comparing 2 practices with identical inclusion criteria differing in third stage management: 2,154 planned, attended low risk vaginal births using expedient squatting to 2,691 planned, attended low risk vaginal births using various forms of active or expectant management.

Main Outcome measures: $\mathrm{PPH} \geq 1000 \mathrm{cc}$ and manual removal of placenta.

Results: Among comparable populations, combinations of active and expectant management resulted in $4.1 \% \mathrm{PPH} \geq 1000 \mathrm{cc}$, whereas expedient delivery of the placenta in standing or squatting by 3 minutes postpartum resulted in $0 \% \mathrm{PPH} \geq 500 \mathrm{cc}$.

Conclusion: Less postpartum bleeding and postpartum hemorrhage occurs when women push out the placenta with the assistance of gravity and expedience, in standing or squatting by 3 minutes after birth.

"The mind of the beginner is empty, open to all possibilities, free of the habits of the expert."

\section{Introduction}

Since the majority of women choosing home birth in high income countries prefer to remain at home postpartum, homebirth midwives are especially motivated to seek ways to avoid postpartum hemorrhage in order to avoid the need for transfer to hospital, Current evidence favored Active Management as resulting in the lowest postpartum hemorrhage rates until the discovery of the expedient squatting protocol at homebirths [1]. The reported $\mathrm{PPH} \geq 1000 \mathrm{cc}$ rates at vaginal birth before and after the adoption of Active management are consistently reported to be 5\%. [2-4] Active Management also increases afterbirth pains, increases the need for postpartum analgesia, increases the incidence of postpartum diastolic blood pressure greater than $90 \mathrm{mmHg}$, and results in increased rehospitalizations due to bleeding. [5]

The main cause of postpartum hemorrhage is uterine atony. An explanation for why uterine atony occurs in humans and not in any other animals is elusive and appears to defy the theory of evolution. Humans share the same placental genetic make-up as apes and chimpanzees who never hemorrhage after birth unless humans interfere with the birth. [6,7] A logical conclusion would be that postpartum hemorrhage has something to do with human intervention. A genetic mutation causing deadly PPH would only be passed on since the relatively recent use of blood transfusions.

Expedient delivery of the placenta allows the now empty uterus to contract quickly, preventing uterine atony, the main cause of PPH. A prospective study of expedient squatting needs very much to be carried out yet this has not taken place. Until such time that there is interest in testing this new protocol, it seems 
fitting to compare delivery of the placenta by 3 minutes postpartum in standing or squatting, to variations of active and expectant third stage management among low risk high income homebirth populations, albeit in different countries, during a similar period.

\section{Methods}

Two different third stage protocols were compared among study and control groups both consisting of women from high income countries with socialized medicine, planning attended homebirth, with similarly trained, licensed, nurse-midwives. PPH was defined as blood loss $\geq 1000 \mathrm{cc}$ in the first 2 hours after birth in both groups. The study group was all births attended from Jan. 1, 2000- Jan 1, 2022 by a Home Birth Service serving a population of approximately 5 million. All midwives working for the Home Birth Service are formally trained and licensed nurse-midwives. Birth outcomes were recorded in a patient record and an Excel spreadsheet immediately following the birth. In the study group, blood loss was estimated to the nearest $10 \mathrm{cc}$. The inclusion criteria for both the study and control groups was identical: Singleton fetus; cephalic presentation; gestational age greater than 36 and less than 41 completed weeks of pregnancy; spontaneous onset of labor; history of up to one previous cesarean; absence of significant pre-existing disease including heart disease, hypertensive chronic renal disease or type 1 diabetes; absence of significant disease arising during pregnancy including hypertension disorders during pregnancy with proteinuria ( $>0.3 \mathrm{~g} / \mathrm{L}$ by urine dipstick), antepartum hemorrhage after 20 weeks' gestation, gestational diabetes requiring insulin, active genital herpes, placenta previa or placental abruption. All study group participants gave written informed consent to deliver the placenta using expedient squatting and to have their outcomes anonymously published. The study group's third stage was managed by: cutting the cord between 1 and 3 minutes after the birth if the woman consented to it, pushing out the placenta in squatting or standing on the floor or in an empty bathtub by 3 minutes; getting into bed by 4 minutes and checking the abdomen to make sure the uterus is contracted at 5 minutes and to massage it to contract if

it is not. In the study group, in the first decade of using the protocol, a prophylactic oral dose of a $0.125 \mathrm{mg}$ pill of Methergine was given between 10 and 20 minutes postpartum to women who had lost $250 \mathrm{cc}$ by that time. Study group blood losses were measured using measuring cups and/or assessing clots. Clots of blood were collected formed into a ball and blood loss calculated according to the calculation for volume of a sphere: $V=(4 / 3) \pi \mathrm{r}: 3 \mathrm{~cm}$ diameter round clot $=14 \mathrm{cc}$ blood loss, $4 \mathrm{~cm}=34 \mathrm{cc} ; 5 \mathrm{~cm}=65 \mathrm{cc}$ blood loss; $6 \mathrm{~cm}=113 \mathrm{cc}, 7 \mathrm{~cm}=180 \mathrm{cc} ; 8 \mathrm{~cm}$ diameter ball $=268 \mathrm{cc}$ blood loss; $9 \mathrm{~cm}=381 \mathrm{cc} ; 10 \mathrm{~cm}=$ $523 \mathrm{cc} ; 12 \mathrm{~cm}=905 \mathrm{cc}$ blood loss. .

Janssen et. al. 2009 [8] was selected out of the high-quality homebirth studies [9] as the control group. Janssen et.al. 2009 overlaps with the study group in time-period (Jan. 1, 2000 to Dec. 31, 2004). Janssen et.al. 2009 is very closely matched in population, birth management, as well as having identical inclusion criteria to the study group. Janssen et.al. was also chosen because it reported the lowest reported PPH rate in the published literature. British Columbia had a similar population to Israel, of 4.4 million at the time of the study. Like in Israel, British Columbian midwifery care is accessible to all women in the province who meet the criteria. The midwives in British Columbia use variations and combinations of active and expectant management. [10] Their database defines PPH as $\geq 1000 \mathrm{~mL}$ blood loss. [11] The 
primary outcome measure was the rate of postpartum hemorrhage $\geq 1000 \mathrm{cc}$. Secondary outcome measure was manual removal of placenta. Formal ethical approval for the methodology of this research received from the Hadassah Medical Organization Helsinki Committee Institutional Review Board, on April 14,2014 . All methods carried out in accordance with guidelines and regulations provided in the ethics approval.

\section{Results}

During the study periods, 2,899 women attended by a registered midwife began labor with the intention of giving birth at home in British Columbia and 2,691 did so, using currently accepted third stage management; and 2,154 women attended by a registered midwife began labor with the intention of giving birth at home in Israel of which 2,149 births did so using expedient squatting or standing to deliver the placenta. Table 1 provides patient demographics and selected outcome measures.

Expedient squatting resulted in no cases of PPH $\geq 1000 \mathrm{cc}$ in the first 2 hours. Variations of active and expectant management resulted in $4.1 \%$ PPH over $1000 \mathrm{cc}$. In the control group, active management resulted in 41 women per 1,000 losing $\geq 1000 \mathrm{cc}$. Expedient squatting resulted in blood losses of an average of $100 \mathrm{cc}$ in the first 2 hours. The one case of PPH over $500 \mathrm{cc}$ was an $800 \mathrm{cc}$ blood loss in a low risk Para 5 woman. The one case of blood transfusion in the study group was another low risk Para 5 woman who began labor with hemoglobin of $7.9 \mathrm{~g} / \mathrm{L}$ and transferred to hospital a day after the birth feeling weak and received one unit of blood. In the control group, two women received blood transfusions. No negative side effects of third stage expedient squatting protocol were observed among 2,149 vaginal births.

\section{Discussion}

In the study population, expedient squatting protocol decreased average postpartum bleeding in the first 2 hours from the current average of $500 \mathrm{cc}$ blood loss at vaginal birth using active management [5] to 100 cc. Expedient squatting decreased $\mathrm{PPH} \geq 1000 \mathrm{cc}$ from $4-0 \%$ and did so without negative side effects or financial expense. This is the first study to report average blood loss of $100 \mathrm{cc}$ in the first hour and / or $0 \%$ $\mathrm{PPH} \geq 1000 \mathrm{cc}$. [12] Other low risk populations report higher PPH rates than Janssen: $4.4 \% \mathrm{PPH} \geq 1000 \mathrm{cc}$. [13] $4.6 \% \mathrm{PPH} \geq 1000 \mathrm{cc}$. [14] $9.3 \% \geq 500 \mathrm{cc}$. [15] and $11.7 \% \mathrm{PPH} \geq 500 \mathrm{cc}$. [16] The two largest high quality homebirth studies: Birthplace and DeJonge et.al. did not report their rates of PPH. $[17,18]$ Large hospital studies looking at multiple sites continue to report 5\% PPH at vaginal birth. [5] Bonding is only interrupted for about one minute that it takes to deliver the placenta in squatting. When relevant, this one minute can be the special time for the partner to hold the newborn baby. As practitioners garnered more experience with expedient squatting, Methergine was no longer needed.

Strengths and limitations: Factors other than timing associated with PPH

The use of IV Pitocin has been associated with PPH but this might be a consequence of factors other than the Pitocin since the PPH rate $\geq 1000 \mathrm{cc}$ prior to the synthesis of Pitocin at vaginal births was the same as 
the PPH rate at vaginal births after the synthesis of Pitocin [3]; and hospital PPH rates in the absence of Pitocin augmentation are about the same as the PPH rates with Pitocin augmentation. [5] In vitro studies show the effect of Pitocin on mouse muscle was attenuated with time. [19-21] This research has been offered as an explanation for the association but fails to explain why a sudden drop in sensitivity, not attenuation, supposedly occurs immediately after birth resulting in PPH. The observation associating Pitocin augmentation with PPH might be due to many possible factors including a delay in delivery of the placenta at births augmented with Pitocin. In the study group, $20 \%$ of labors were not augmented with IV Pitocin but rather with bilateral nipple massage. Nipple massage, if done correctly, causes contractions by releasing Oxytocin. It is unknown if nipple massage has any association with $\mathrm{PPH}$.

Both nulliparity and grand multiparity are associated with higher rates of PPH [5]. The study group had fewer nulliparas: $26 \%$ vs. $44 \%$ and likely more grand multiparous women. Although the control group did not report grand multiparity, it is likely the $23 \%$ study group rate for grand multiparous women (P6-P12) is higher than that of British Columbia, as the fertility rate in Israel is higher than in British Columbia. $0 \% \mathrm{PPH}$ was found among the grand multiparas (P6 -P12) who delivered using expedient squatting. The findings here suggest the associations between $\mathrm{PPH}$ and nulliparity or grandmultiparity disappear when the placenta is delivered by 3 minutes in squatting.

A common critique of studies reporting postpartum blood loss is underreporting PPH. Underreporting of $\mathrm{PPH}$ is suspected even in the highest quality studies. [5] The best definition of PPH is a larger than $20 \mathrm{~g} / \mathrm{L}$ drop in hemoglobin level compared to the level before the birth. This, however, requires reliable blood testing before and after the birth. Erickson et.al. [4] suggests that the terms PPH over $500 \mathrm{cc}$ and PPH over $1000 \mathrm{cc}$ are indistinguishable without hemoglobin blood level testing [page 610] since both look very similar on disposable 60X90 pads. It is indeed reasonable to suggest that blood losses between 500 and $1000 \mathrm{cc}$ are indistinguishable. However, this observation is not relevant to the results of this study because the average blood loss was significantly less than $500 \mathrm{cc}$. It is impossible (for the non-visually impaired) to confuse $100 \mathrm{cc}$ blood loss with blood loss of $500 \mathrm{cc}$ at birth, just as it is impossible to confuse $100 \mathrm{cc}$ urine in a Foley bag with $500 \mathrm{cc}$ of urine. The typical $100 \mathrm{cc}$ blood loss consists of a tablespoon or $15 \mathrm{cc}$ attached to the placenta, another $30 \mathrm{cc}$ on the $60 \times 90$ pad she gives birth on, and $50 \mathrm{cc}$ seen 2 hours after birth on the pad used starting from 5 minutes after birth when she got into bed.

Interpretation

Except for very exceptional cases, women appear not to experience PPH of over $500 \mathrm{cc}$ where the placenta is delivered expediently in standing or squatting.

\section{Conclusion}

Expedient Squatting Protocol appears to be a simple way to use gravity and expedience to prevent postpartum hemorrhage. It's an invention that causes the user to wonder how the idea wasn't thought of years ago. 


\section{Declarations}

- Ethics approval and consent to participate- Ethics Approval from Hadassah Medical Center Helsinki Committee on April 14, 2014. Signed, Written, Informed consent to participate was received from each woman in the study group.

Guidelines statement. All methods were carried out in accordance with relevant guidelines and regulations. All experimental protocols were approved by the named licensing committee. The experiments were performed in accordance with relevant guidelines and regulations.

Consent for publication- Not applicable. There is no information or images that could lead to identification of a study participant. The initial quality checker acknowledged that in this manuscript there are no such images or identifiable information given.

Availability of data and materials: This study is elegantly simple and easy to reproduce. All relevant data generated during this study are included in this published article.

Competing interests-While the authors have no competing interests, it might be stated that if this COSTFREE, LIFE SAVING protocol with absolutely no negative side effects is not accepted for publication, then the editors and peer reviewers have a competing interest to PROMOTE, rather than reduce or eliminate postpartum hemorrhage. To date, no worthy explanation has been provided for not publishing this protocol.

Funding_all expenses including the cost of publication paid for by the corresponding author.

Authors' contributions- JSC and REB shared the work equally.

Acknowledgements-Many thanks to the women for squatting out their placentas expediently.

\section{References}

1. Cohain JS. A Proposed Protocol for Third Stage Management- Judy's 3,4,5,10 minute method. Birth. 2010: 37(1)84-5.

https://www.researchgate.net/publication/43200173_A_proposed_protocol_for_third_stage_management? ev=prf_pub

2. Melody GF. Primary postpartum hemorrhage. Calif Med. 1951;75(6):425-429.

3. Magann EF, Evans S,Chauhan SP et.al.The length of the third stage of labor and the risk of postpartum hemorrhage.Obstet Gynecol. 2005;105(2):290-3.

4. Erickson EN, Lee CS, Carlson NS. Predicting Postpartum Hemorrhage After Vaginal Birth by Labor Phenotype. J Midwifery Womens Health. 2020;65(5):609-620. 
5. Begley CM, Gyte GML, Devane D et.al. Active versus expectant management for women in the third stage of labour. Cochrane Database of Systematic Reviews 2019,Issue 2. Art. No.: CD007412

6. Abrams ET, Rutherford JN. Framing postpartum hemorrhage as a consequence of human placental biology: an evolutionary and comparative perspective. Am Anthropol. 2011. 113, 3, 417-30.

7. Carter AM, Pijnenborg R. Evolution of invasive placentation with special reference to non-human primates. Best Pract Res Clin Obstet Gynaecol. 2011;25(3):249-57.

8. Janssen PA, Saxell L, Page LA et.al. Outcomes of planned home birth with registered midwife versus planned hospital birth with midwife or physician. CMAJ 2009;181(6-7):377-383.

9. Cohain JS. Instead of attacking homebirth, hospital practitioners should be trying to understand why their outcomes compared unfavorably to homebirth outcomes. BJOG. 2016;123(7):1231.

https://www.researchgate.net/publication/303412758_Instead_of_attacking_home_birth_hospital_ practitioners_should_be_trying_to_understand_why_their_outcomes_compare_unfavourably_ with_home_birth_outcomes

10. Tan WM, Klein MC, Saxell L, Shirkoohy SE, Asrat G. How do physicians and midwives manage the third stage of labor? Birth. 2008;35(3):220-9.

11. Janssen PA, Lee SK, Ryan EM et.al. Outcomes of planned home births versus planned hospital births after regulation of midwifery in British Columbia. CMAJ. 2002;166(3):315-323.

12. Fahy KM. Third stage of labour care for women at low risk of postpartum haemorrhage. J Midwifery Womens Health 2009;54(5): 380-386.

13. Janssen PA, Lee SK, Ryan EM et.al. Outcomes of planned home births versus planned hospital births after regulation of midwifery in British Columbia. CMAJ. 2002;166(3):315-323.

14. Bolten $\mathrm{N}$, de Jonge $\mathrm{A}$, Zwagerman $\mathrm{E}$ et.al. Effect of planned place of birth on obstetric interventions and maternal outcomes among low-risk women: a cohort study in the Netherlands. BMC Pregnancy Childbirth. 2016;16(1):329.

15. Hutton EK, Reitsma AH, Kaufman K. Outcomes associated with planned home and planned hospital births in low-risk women attended by midwives in Ontario, Canada, 2003-2006: a retrospective cohort study. Birth. 2009;36(3):180-189.

16. Campiotti M, Campi R, Zanetti M et.al. Low-Risk Planned Out-of-Hospital Births: Characteristics and Perinatal Outcomes in Different Italian Birth Settings. Int J Environ Res Public Health. 2020;17(8):2718.

17. Birthplace in England Collaborative Group, Brocklehurst P, Hardy P et.al. Perinatal and maternal outcomes by planned place of birth for healthy women with low risk pregnancies: the Birthplace in England national prospective cohort study. BMJ. 2011;343:d7400. 
18. de Jonge A, Peters L, Geerts CC et.al. Mode of birth and medical interventions among women at low risk of complications: A cross-national comparison of birth settings in England and the Netherlands. PLoS One. 2017;12(7):e0180846.

19. Balki M, Ramachandran N, Lee S, Talati C. The recovery time of myometrial responsiveness after oxytocin-induced desensitization in human myometrium in vitro. Anesth Analg. 2016;122(5):1508-1515.

20. Balki M, Erik-Soussi M, Kingdom J, Carvalho J. Oxytocin pretreatment attenuates oxytocin-induced contractions in human myometrium. Anesthesiology. 2013;119(3):552-561.

21. Talati C, Carvalho JCA, Luca A, Balki M. The effect of intermittent oxytocin pretreatment on oxytocininduced contractility of human myometrium in vitro. Anesth Analg. 2019;128(4):671-678.

\section{Table}

Table 1: Patient Demographics and Outcomes 


\begin{tabular}{|c|c|c|}
\hline Age & Control Group & Study Group \\
\hline Maternal Age 15-19 & $2 \%$ & $0.40 \%$ \\
\hline Maternal Age 20-24 & $12 \%$ & $6 \%$ \\
\hline Maternal Age 25-29 & $31 \%$ & $30 \%$ \\
\hline Maternal Age 30-34 & $35 \%$ & $39 \%$ \\
\hline Maternal Age 35 or over & $21 \%$ & $24 \%$ \\
\hline Single Parent & $3 \%$ & $3 \%$ \\
\hline Average BMI & 22.8 & 22.4 \\
\hline Illicit Drug Use during Pregnancy & $1 \%$ & $0.40 \%$ \\
\hline Smoking during Pregnancy & $6 \%$ & $0.20 \%$ \\
\hline Vaginal Birth & $93 \%$ & $99 \%$ \\
\hline Nulliparous & $42 \%$ & $26 \%$ \\
\hline Multiparous & $58 \%$ & $74 \%$ \\
\hline GrandMultiparous: P6-12 & Not reported & $23 \%$ \\
\hline Medical Induction & 0 & 0 \\
\hline Augmentation of Labor & $24 \%$ & $20 \%$ \\
\hline Amniotomy & $19 \%$ & $8 \%$ \\
\hline Uterotonic drug postpartum & $6 \%$ & 5\% PO Methergine \\
\hline Epidural & $5 \%$ & $0 \%$ \\
\hline Narcotic Analgesic pain relief & $4 \%$ & $50 \%$ PO Rokacet \\
\hline Vaginal & $90 \%$ & $99 \%$ \\
\hline Assisted & $3 \%$ & $0.10 \%$ \\
\hline Cesarean & $7 \%$ & $0.30 \%$ \\
\hline Episiotomy among vaginal deliveries & $3 \%$ & 0 \\
\hline First or second degree tear & $44 \%$ & $3 \%$ \\
\hline Third or Fourth degree tear & $1 \%$ & 0 \\
\hline Cervical tear & $0.10 \%$ & $0.10 \%$ \\
\hline Blood Transfusions & $2 / 2691$ & $1 / 2149$ \\
\hline Obstetric shock & $1 / 2691$ & $0 / 2149$ \\
\hline
\end{tabular}


